1. Mrug M, Mishra PV, Lusane HC, Cunningham JM, Alpert MA Hemothorax and retroperitoneal hematoma after anticoagulation with enoxaparin. South Med J 2002; 95: 936-8.

2. Prasad S, Patankar T, Krishnan A, Pathare A. Spontaneous isolated lesser sac hematoma in a patient with hemophilia. Indian J Gastroenterol 1999; 18: 38-9.

3. Skudder PA Jr, Craver WL. Mesenteric hematoma suggests rupture of visceral artery aneurysm. Arch Surg 1984; 119: 863.

4. García Florez L, Martínez Rodríguez E. Spontaneous hematoma of the transverse mesocolon. Rev Esp Enferm Dig 1993; 83: 130-2.

5. Carr SC, Pearce WH, Voguelzang RL, et al. Current management of visceral artery aneurysms. Surgery 1996; 120: 627-33.

6. Kabaroudis A, Papaziogas B, Papaziogas T. Spontaneous retroperitoneal hematoma caused by aneurysm of the inferior pancreaticoduodenal artery. Am J Surg 2002; 184: 174-5.

7. Raghavendra BN, Grieco AJ, Balthazar EJ, Megibow AJ, Subramanyam BR. Diagnostic utility of sonography and computed tomography in spontaneous mesenteric hematoma. Am J Gastroenterol 1982; 77: $570-3$

\section{Doble rotura cardiaca post-infarto agudo de miocardio}

\section{Sr. Director:}

La CIV post-infarto agudo de miocardio (IAM) antes de la era de la reperfusión se presentaba en el 1 a $3 \%$ de todos los IAM (1). Sin reperfusión previa como es nuestro caso la rotura septal ocurre generalmente dentro de la primera semana. La mortalidad de la CIV post IAM con tratamiento conservador es $24 \%$ en las primeras 24 horas; $46 \%$ en la primera semana y $70-80 \%$ a los dos meses (3-5). Según Lemery (2) 24\% de supervivencia a los 30 días con tratamiento conservador y $47 \%$ de supervivencia con cirugía. A continuación se expone el caso de un paciente que tiene como interés una supervivencia de tres años después de la reparación quirúrgica de una inhabitual doble rotura cardiaca sin reperfusión previa ya que acudió varios días después de la fase aguda de infarto.

Se trata de un varón de 65 años de edad con antecedentes de tabaquismo importante, criterios de bronquitis crónica y diabetes mellitus tipo II que acude al servicio de urgencias por disnea, ortopnea y edemas importantes de extremidades inferiores de aproximadamente 10 días de evolución. La exploración física es compatible con una situación de insuficiencia cardiaca derecha con estasis venoso yugular, hepatomegalia de $10 \mathrm{~cm}$. con maniobra de reflujo positivo y edemas de extremidades inferiores, llamando la atención un soplo sistólico grado III/IV en BEI. La glucemia es de $118 \mathrm{mg} / 100 \mathrm{ml}$. CPK 82 UI. LDH 538 UI. Troponina T 0,22. ECG: Ritmo sinusal con ondas P de dilatación auricular derecha y ondas Q en V1 y V2 La radiografía de tórax muestra ligera cardiomegalia, hipertensión pulmonar y ligero edema pulmonar intersticial. El ecocardiograma presenta CIV a nivel medio posterior de tamaño importante con shunt izquierdo-derecho; aneurisma postero-inferior y de septo de gran tamaño con importante disquinesia; hipertensión pulmonar severa estimada en 70 $\mathrm{mm}$. de $\mathrm{Hg}$ ventrículo izquierdo ligeramente dilatado con función globalmente deprimida y ventrículo derecho ligeramente dilatado con buena contractilidad. La coronariografía aporta imagen de lesión severa proximal de diag 1 de la descendente anterior y de

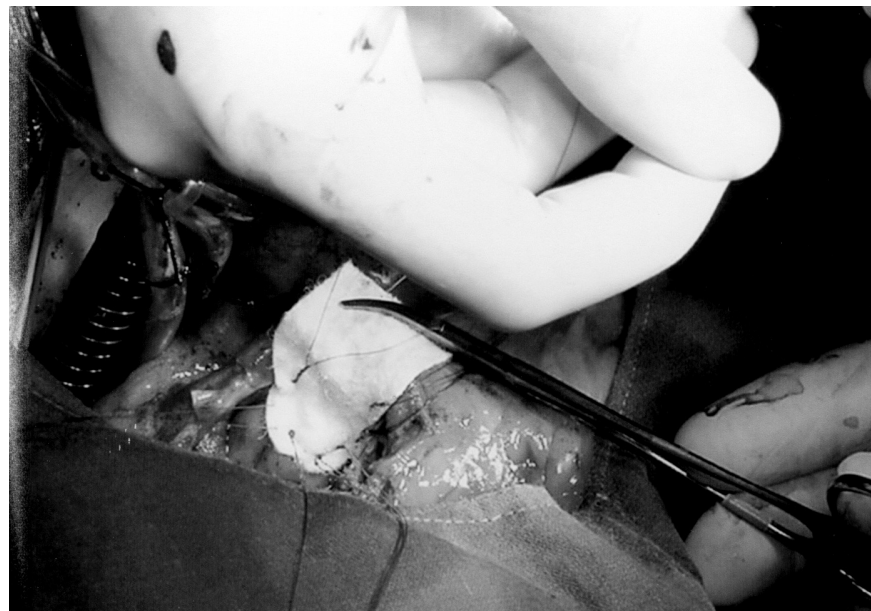

Fig. 1. Cierre de CIV con parche de teflón.

OM 1 de la circunfleja así como coronaria derecha ateromatosa con oclusión distal no visualizándose lecho distal ni por circulación colateral. No se realiza reperfusión previa a cirugía dado lo diferido del episodio. Como hallazgo intraoperatorio además de la CIV ya referida la presencia de rotura contenida del aneurisma por trombo intramural. Se realiza cierre de CIV con parche de teflón, plicatura de aneurisma con técnica de Dor y cierre de rotura taponada de aneurisma con parche de teflón y refuerzo de la rotura.

El paciente actualmente después de tres años vive en situación de insuficiencia cardíaca grado II-III de la NYHA. Esta evolución favorable sería sorprendente si no se conociera que previamente este paciente había sobrevivido a un carcinoma bronquial T2 N0 M0. Posteriormente un episodio de hemiparesia izquierda de predominio braquial con TAC craneal en el que se apreciaba una tumoración parietal derecha única metastásica que cura con cirugía esterotáxica más radioterapia. Diverticulitis aguda complicada con obstrucción intestinal, un año después, encontrándose en la cirugía de la reconstrucción una lesión proliferativa neoplásica a $8 \mathrm{~cm}$. de margen anal. Un ejemplo de supervivencia a diferentes procesos todos y cada uno de ellos con alta mortalidad.

\section{F. J. Cabrera Aguilar, A. Muiño Miguez, B. Pinilla Llorente, E. Lopez de Sá Areses ${ }^{1}$, J. Silva Guisasola ${ }^{2}$}

Servicios de Medicina Interna, ${ }^{1}$ Cardiología ${ }^{2}$ Cirugía Cardiaca. Hospital General Universitario Gregorio Marañón. Madrid

1. Moore CA, Nygaard TW, Kaiser DL, Cooper AA, Gibson RS. Postinfarction ventricular septal rupture: the importance of location of infarction and right ventricular function in determining survival. Circulation 1986; 74: 45-55.

2. Lemery R, Smith HC, Giuliani ER, Gersh BJ. Prognosis in rupture of the ventricular septum after acute myocardial infarction and role of early surgical intervention. Am J Cardiol 1992; 70:147-51.

3. Birbaum Y, Fishbein M, Blanche C, Siegel R. Ventricular septal rupture after acute myocardial infarction. N Eng J Med 2002; 347: 1426: 32.

4. Sugiura T, Nagahama Y, Nakamura S, Kudo Y, Yamasaki F, Iwasaka T. Left ventricular free wall rupture after reperfusion therapy for acute myocardial infarction. Am J Cardiol 2003; 92: 282-4.

5. Wehrens X, Doevendans P. Cardiac rupture complicating myocardial infarction. Int J Cardiol 2004; 95: 285-92. 